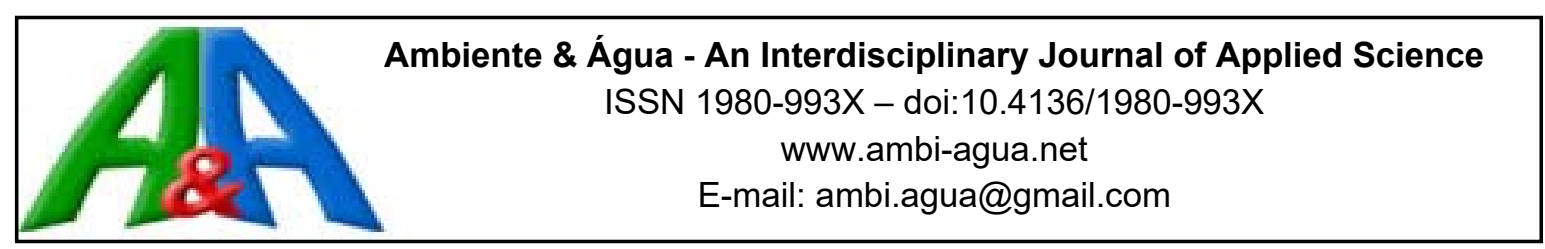

\title{
Validação de método analítico e de extração do malation em água e solo após nebulização de combate ao Aedes aegypti
}

doi:10.4136/ambi-agua.1993

Received: 27 Aug. 2016; Accepted: 22 Mar. 2017

\author{
Ana Carla Coleone ${ }^{1,2^{*}}$; Flavio Soares da Silva ${ }^{3}$; Angela Aparecida Machado ${ }^{2}$; \\ Joaquim Gonçalves Machado Neto ${ }^{2}$; Wanderley da Silva Paganini ${ }^{1}$ \\ ${ }^{1}$ Universidade de São Paulo (USP), São Paulo, SP, Brasil \\ Departamento de Saúde Ambiental \\ ${ }^{2}$ Universidade Estadual Paulista (UNESP), Jaboticabal, SP, Brasil \\ Departamento de Fitossanidade \\ ${ }^{3}$ Universidade Federal de Itajubá (UNIFEI) Itajubá, MG, Brasil \\ *Autor correspondente: e-mail: anacarlacb@yahoo.com.br, \\ flavio_ss_ss@yahoo.com.br, angela_machado88@hotmail.com, \\ joaquim@fcav.unesp.br, paganini@usp.br
}

\section{RESUMO}

O mosquito Aedes aegypti é vetor de doenças de grande relevância para a saúde pública, como a dengue, febre amarela, zika e chikungunya. Em áreas urbanas, para o controle dos insetos adultos, é realizada a nebulização a ultrabaixo volume (UBV) do malation. A pulverização pode levar à contaminação ambiental do solo e da água com o inseticida. Objetivou-se ajustar e validar um método analítico para determinação de malation em cromatografia líquida de alta eficiência (CLAE) e determinar o método de extração do inseticida das matrizes água e solo. A fase móvel foi uma mistura isocrática de acetonitrila e água (68:32), à vazão de $1 \mathrm{~mL}$ min $^{-1}$, o volume de injeção de $20 \mu 1 \mathrm{e}$ UV a $210 \mathrm{~nm}$. Após fortificação, o analito foi extraído com acetonitrila (ACN) por agitação mecânica nas amostras de solo, e por evaporação das amostras de água, pré-concentrado, redissolvido em ACN e a recuperação foi calculada. O método é simples, específico e adequado para análise do malation com linearidade entre as concentrações de 0,05 e $1,00 \mu \mathrm{g} \mathrm{mL} \mathrm{m}^{-1}\left(\mathrm{R}^{2}=0,999\right)$ e limites de

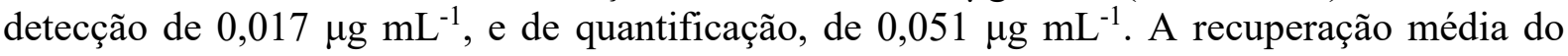
malation da água foi de $97 \%$ e do solo, $96 \%$, sendo o método exato e preciso (coeficiente de variação $<8,8 \%$ ). O método ajustado pode ser utilizado com segurança para quantificação do inseticida em matrizes de água e solo.

Palavras-chave: cromatografia líquida, inseticida, saúde pública.

\section{Analytical method of validation and extraction of malathion in water and soil after fogging against Aedes aegypti}

\begin{abstract}
The Aedes aegypti mosquito is a vector of diseases such as dengue, yellow fever, chikungunya and zika, which has great relevance to public health. In urban areas, ultra-low volume (ULV) malathion fogging is used for the control of adult mosquitoes. Spraying with the insecticide can contaminate soil and water. The goal of this study was to adjust and validate an
\end{abstract}


analytical method for the determination of malathion in high-performance liquid chromatography (HPLC) and determine the pesticide extraction method of water and soil matrices. The mobile phase used an isocratic mixture of acetonitrile and water (68:32), at a flow rate of $1 \mathrm{~mL} \mathrm{~min}^{-1}$, an injection volume of $20 \mu \mathrm{L}$, and a UV at $210 \mathrm{~nm}$. After fortification, the analyte was extracted with acetonitrile $(\mathrm{ACN})$ by mechanical agitation of the soil samples, and by evaporation of the water samples, pre-concentrated, re-dissolved in ACN, and the recovery was calculated. The method is simple, specific and suitable for analysis of malathion with linearity between concentrations of 0.050 and $1.00 \mu \mathrm{g} \mathrm{mL}^{-1}\left(\mathrm{R}^{2}=0.999\right)$ and detection limits of $0,017 \mu \mathrm{g} \mathrm{mL}^{-1}$ and quantification of $0.051 \mu \mathrm{g} \mathrm{mL} \mathrm{L}^{-1}$. The average recovery of malathion from water was $97 \%$, and from soil, $96 \%$, being the method exact and precise (coefficient of variation $<8.8 \%$ ). The set method can be safely used for the quantification of the insecticide in water and soil matrices.

Keywords: insecticide, liquid chromatography, public health.

\section{INTRODUÇÃO}

O mosquito Aedes aegypti é um dos maiores problemas da Saúde Pública nas regiões tropicais e subtropicais do mundo. Além de ser o principal vetor da dengue e da febre amarela em áreas urbanas no Brasil, recentemente foi identificado também como transmissor da zika e da chikungunya, arboviroses responsáveis por sérias disfunções ou malformações.

Em áreas de grande transmissão de doenças ocasionadas por esse vetor, é realizado o controle químico do inseto com a nebulização do inseticida organofosforado malation, aplicado com turbo atomizado, calibrado com volume de aplicação ultrabaixo volume (UBV) a frio, conforme orientação da Organização Mundial da Saúde (OMS) e do Ministério da Saúde (MS).

No Brasil, esta prática é realizada desde a década de 1960, com a aplicação do malation diluído em óleo de soja, e mais recentemente, em água. Para a nebulização são utilizados equipamentos de transporte costal ou acoplados a veículos, com aplicação nas vias públicas, terrenos, parques e praças, ou no interior das edificações (Brasil, 2009).

O controle vetorial a UBV consiste na fragmentação de uma pequena quantidade do inseticida pelo equipamento de aplicação, formando aerossóis. Parte destes aerossóis sofre deriva, podendo depositar-se sobre o solo e nas águas locais (Chaim, 1989). Portanto, ajustar métodos químicos analíticos e determinar resíduos do malation nas matrizes água e solo é de extrema importância para monitorar a contaminação ambiental devido ao combate ao A. aegypti com este inseticida, cujos efeitos tóxicos ao homem e demais organismos são comprovados por muitos pesquisadores.

A determinação de resíduos de pesticidas em diferentes matrizes é tradicionalmente realizada por meio de técnicas cromatográficas, devido à facilidade de separação, identificação e quantificação dos analitos presentes na amostra, e há diversos detectores com possibilidade de uso.

A cromatografia a gás $(\mathrm{CG})$ é uma técnica muito utilizada na quantificação de compostos organofosforados. Contudo, alguns autores descrevem métodos de análise por cromatografia líquida de alta eficiência (CLAE). Em análise de malation em plasma e urina, Abu-Qare e AbouDonia (2001) utilizaram um detector de ultravioleta (UV) a $210 \mathrm{~nm}$ e a fase móvel composta por acetonitrila e água. Em matriz água, Morales et al. (2012) utilizaram CLAE com detector de arranjo de diodos, e metanol e água como fase móvel. Kulluru et al. (2010) também utilizaram CLAE para análise de malation em solo, com fase móvel consistindo de acetonitrila e água e detector de UV a $254 \mathrm{~nm}$.

Para quantificação de um pesticida em determinada matriz é necessária a validação do método analítico, que consiste na determinação de alguns parâmetros como especificidade, 
linearidade, limites de detecção e quantificação, exatidão e precisão dos dados, como recomendado pela Resolução $n^{\circ} 899$ da Agência Nacional de Vigilância Sanitária - ANVISA (Anvisa, 2003) e pela NBR14029:2005 da ABNT (ABNT, 2005).

Ensaios de recuperação são normalmente utilizados para validação do método analítico a fim de estimar-se a exatidão e a precisão dos dados. Estes ensaios são realizados com a fortificação da matriz, ou seja, com adição de uma concentração conhecida do analito à amostra. Segundo Brito et al. (2003), a recuperação consiste na relação percentual entre a concentração real e a concentração determinada após aplicação do procedimento analítico, e é calculada para diferentes níveis de fortificação. A porcentagem adequada de recuperação de um analito é estabelecida em função de sua concentração, conforme as classes descritas pela Association of Official Analytical Chemists (AOAC, 1998).

A existência de interferentes nas matrizes e a presença das substâncias de interesse em baixas concentrações requerem a utilização de técnicas de extração, purificação no caso de amostras muito complexas, e pré-concentração para que a determinação dos componentes de interesse se torne possível. O objetivo final é a obtenção de uma subfração da amostra original limpa e enriquecida com as substâncias de interesse analítico. Uma maneira pouco custosa de pré-concentrar uma amostra é a evaporação seguida da redissolução do analito em um pequeno volume de solvente.

A extração de amostras livres de interferentes que prejudiquem a análise cromatográfica, como determinadas amostras de água, pode ser feita simplesmente com a evaporação e redissolução do composto de interesse em solvente específico (Amarante et al., 2002). Para a extração de pesticidas de matrizes mais complexas como o solo, os métodos mais frequentemente empregados são o Soxhlet, agitação mecânica e banhos ultrassônicos com solventes orgânicos como acetonitrila, hexano, diclorometano, acetona e acetato de etila (Bernal et al., 1996; Tahir et al., 2010).

O objetivo deste estudo foi ajustar e validar o método analítico para análise de malation em CLAE e determinar o método de extração do inseticida das matrizes água e solo.

\section{MATERIAL E MÉTODOS}

\subsection{Local de estudo}

Os estudos foram realizados no Laboratório de Ecotoxicologia dos Agrotóxicos e Saúde Ocupacional do Departamento de Fitossanidade da Faculdade de Ciências Agrárias e Veterinárias da Universidade Estadual Paulista (UNESP), campus de Jaboticabal.

\subsection{Reagentes e soluções}

Para validação do método cromatográfico em água e solo, foi utilizado o padrão analítico de malation (grau PESTANAL ${ }^{\circledR}$ ), com $97,5 \%$ de pureza. A acetonitrila utilizada foi de grau CLAE, da JT BAKER ${ }^{\circledR}$. A água ultrapura empregada na fase móvel foi obtida em equipamento Elga, PurelabUHQ.

As soluções estoques de malation para elaboração da curva analítica foram preparadas diluindo o padrão analítico em acetonitrila, em uma concentração de $1000 \mu \mathrm{g} \mathrm{mL}^{-1}$. Diluições sucessivas foram realizadas obtendo-se as concentrações de 0,$05 ; 0,10 ; 0,25 ; 0,50 ; 0,75$ e 1,00 $\mu \mathrm{g} \mathrm{mL} \mathrm{m}^{-1}$ de malation.

\subsection{Equipamentos}

A pesagem do padrão analítico foi realizada em balança analítica Sartorius, BL210S e do solo, em balança semianalítica Bel, P236. 
Para elaboração das soluções estoque e procedimentos de fortificação e extração do malation nas diferentes matrizes, foram utilizadas micropetas automáticas da marca Gilson e vidraria volumétrica adequada.

Um sistema de evaporador rotativo Fisaton, 802A, acoplado a um banho ultrassônico e um agitador de tubos Phoenix, YAP56, foram utilizados na extração do inseticida do solo e da água. Uma agitadora Tecnal, TE 421 foi empregada na extração do malation do solo.

A análise do inseticida foi realizada em sistema de cromatografia líquida de alta eficiência (CLAE) Surveyor LC ThermoFinnigan ${ }^{\circledR}$ acoplado a um detector UV visível (software Chromquest 4.1).

Toda a vidraria utilizada neste estudo foi higienizada de acordo com o preconizado pela NBR 13073 (ABNT, 2003).

\subsection{Validação do método analítico do malation por CLAE}

\subsubsection{Condições cromatográficas}

O método analítico foi adaptado do método descrito por Abu-Qare e Abou-Donia (2001). O sistema cromatográfico adotado foi composto por duas fases móveis 68:32 (v:v): acetonitrila grau CLAE (fase A) e água ultrapura (fase B), filtradas em membrana de $0,45 \mu \mathrm{m}$ e desgaseificadas em ultrassom por 15 minutos.

A vazão e o volume de injeção utilizados foram, respectivamente, $1 \mathrm{~mL} \mathrm{~min}^{-1} \mathrm{e} 20 \mu \mathrm{L}$ e o comprimento de onda utilizado no detector de ultravioleta (UV-visível) foi de $210 \mathrm{~nm}$. A coluna empregada foi ACE C18 $(250 \times 4,6 \mathrm{~mm})$ e $5 \mu \mathrm{m}$ de tamanho de partícula.

\subsubsection{Validação do método}

A validação do método analítico foi realizada após injeção de sete réplicas (NBR14029:2005; ABNT, 2005) de cada concentração de estudo para os parâmetros especificidade, linearidade, limites de detecção e quantificação e cinco réplicas (Resolução $n^{\circ}$ 899 da ANVISA; Brasil, 2003) para exatidão e precisão.

A curva analítica foi elaborada com concentrações variando de $0,05-1,00 \mu \mathrm{g} \mathrm{mL}^{-1}$, caracterizando o intervalo de trabalho, ou seja, a faixa da menor à maior concentração que pode ser determinada com precisão e exatidão em relação à linearidade e acima do limite de quantificação obtido pelo método.

Considerou-se a região linear, aquela em que o resultado obtido foi proporcional à concentração do analito. Para avaliação da linearidade, foi dividido o fator de resposta (área média/concentração) pela concentração. As áreas médias/concentração foram ordenadas e a mediana (independente dos valores anômalos) foi determinada com 10\% de variação superior e inferior.

A especificidade foi analisada para verificação da existência de interferência de compostos das matrizes na análise do inseticida.

O limite de detecção (LD), considerado como a menor concentração que pode ser detectada, mas não quantificada, e o limite de quantificação (LQ), considerado como a menor concentração que pode ser quantificada com exatidão e precisão aceitáveis, foram calculados a partir dos parâmetros da curva analítica das Equações 1 e 2.

$$
\begin{aligned}
& \mathrm{LD}=3,3 \times \frac{s d}{b} \\
& \mathrm{LQ}=3 \times L D
\end{aligned}
$$

em que:

$\mathrm{Sd}=$ desvio padrão da curva analítica, e 
$b=$ coeficiente angular da curva analítica.

A exatidão e a precisão intraensaio foram calculadas com três concentrações: no início $\left(0,05 \mu \mathrm{g} \mathrm{mL}^{-1}\right)$, meio $\left(0,50 \mu \mathrm{g} \mathrm{mL}^{-1}\right)$ e fim da curva analítica $\left(1,00 \mu \mathrm{g} \mathrm{mL}{ }^{-1}\right)$. Para o cálculo da exatidão, utilizou-se o valor da recuperação das amostras. Já a precisão foi calculada pela dispersão dos resultados e expressa pelo coeficiente de variação dos dados obtidos.

\subsection{Método de extração do malation das matrizes de água e solo}

\subsubsection{Fortificação e extração das amostras}

Testes de fortificação e recuperação do malation em $10,00 \mathrm{~mL}$ de água e $10,00 \mathrm{~g}$ de solo argiloso foram realizados em sala climatizada a $20 \pm 5^{\circ} \mathrm{C}$, a fim de verificar-se a porcentagem de recuperação do analito após o processo de pré-concentração e extração das matrizes. $\mathrm{O}$ volume de água e a massa de solo escolhidos neste estudo ocorreu para que a pré-concentração satisfizesse a quantificação do inseticida nas amostras de campo acima do limite de quantificação após a pulverização do inseticida na dose de campo indicada pelo Ministério da Saúde de $146 \mathrm{~g} \mathrm{ha}^{-1}$ (Brasil, 2009).

A água utilizada nestes testes foi proveniente de sistema público de abastecimento, sem passar por processo de destilação, filtrada em filtro de $0,45 \mu \mathrm{m}$ e livre da contaminação por malation, como indicaram as análises cromatográficas prévias desta matriz. O mesmo foi observado para o solo, predominantemente argiloso (57\% de argila), obtido da camada de 0-20 $\mathrm{cm}$ de profundidade do campo da Fazenda Experimental da Faculdade de Ciências Agrárias e Veterinárias de Jaboticabal.

As amostras foram fortificadas adicionando-se $1 \mathrm{~mL}$ de padrão analítico de 0,$05 ; 0,5 \mathrm{e}$ $1,0 \mu \mathrm{g} \mathrm{mL} \mathrm{m}^{-1} \mathrm{em} 9 \mathrm{~mL}$ de água, correspondentes a três níveis da curva padrão: o mais baixo, um médio e o maior, obtendo-se dessa maneira, concentrações de $0,005 \mu \mathrm{g} \mathrm{mL}{ }^{-1} ; 0,05 \mu \mathrm{g} \mathrm{m} \mathrm{L}^{-1} \mathrm{e}$ $0,1 \mu \mathrm{g} \mathrm{mL} \mathrm{L}^{-1}$.

As amostras de solo foram umidificadas com $1 \mathrm{~mL}$ de acetonitrila e então fortificadas. Após este processo foram vigorosamente homogeneizadas manualmente e em agitadora por 10 minutos, sendo então deixadas em repouso por 2 horas no escuro, com ventilação constante a $20^{\circ} \mathrm{C}$ para eliminação do solvente. A metodologia utilizada neste processo foi baseada principalmente no trabalho de Bernal et al. (1996) para extração de herbicidas.

Em seguida, volumes de $30 \mathrm{~mL}$ de acetonitrila foram adicionados às amostras, que foram agitadas por 2 horas. O solvente, por sua vez, foi coletado e filtrado em filtro de $0,45 \mu \mathrm{m}$, dentro de balão de fundo redondo. Mais $20 \mathrm{~mL}$ de acetonitrila foram adicionados às amostras de solo, que seguiram para agitação por mais 30 minutos, material posteriormente coletado e devidamente filtrado.

Os balões contendo as amostras seguiram para rotoevaporação a $40 \mathrm{rpm}$ nas mesmas condições das amostras de água, em evaporador rotativo, cujo banho de aquecimento foi de $60^{\circ} \mathrm{C}$ e o banho ultratermostatizado, a $4^{\circ} \mathrm{C}$, até a evaporação total da solução, quando foi removido do sistema e deixado em repouso até resfriamento à temperatura ambiente.

Então, $1 \mathrm{~mL}$ de acetonitrila foi adicionado ao balão, que seguiu para um banho ultrassônico por 10 minutos. Após esta etapa, o balão foi agitado por mais 2 minutos em agitador de tubos. Em seguida, a amostra foi coletada e transferida para vial, seguindo para análise em CLAE.

\subsubsection{Recuperação das amostras}

A recuperação do analito das amostras de água e solo foi calculada segundo a seguinte fórmula da Equação 3. 


$$
\% \operatorname{Rec}=\frac{\text { Concobs }}{\text { Concexp }} \times 100
$$

em que:

$\% \operatorname{Rec}=$ recuperação percentual do analito obtido no ensaio de recuperação,

Concobs $=$ concentração observada do analito na solução contaminada, e

Concexp $=$ concentração esperada do analito na solução contaminada.

A recuperação para as três concentrações de estudo foi verificada de acordo com a faixa recomendada pela Association of Official Analytical Chemists (AOAC, 1998). Segundo a AOAC, o nível aceitável para recuperação de agrotóxicos em diferentes matrizes depende da concentração investigada. Os valores aceitos encontram-se na Tabela 1.

Tabela 1. Nível aceito de recuperação de um analito em função da sua concentração.

\begin{tabular}{lc}
\hline $\begin{array}{c}\text { Concentração do analito } \\
(\%)\end{array}$ & $\begin{array}{c}\text { Intervalo de } \\
\text { recuperação aceito } \\
(\%)\end{array}$ \\
\hline$\geq 10$ & $98-102$ \\
$\geq 1$ & $97-103$ \\
$\geq 0,1$ & $95-105$ \\
$\geq 0,01$ & $90-107$ \\
$\geq 0,001->0,0001$ & $80-110$ \\
$\geq 0,000001$ & $60-115$ \\
$\geq 0,0000001$ & $40-120$ \\
\hline
\end{tabular}

Fonte: Brito et al. (2003).

\section{RESULTADOS E DISCUSSÃO}

\subsection{Validação do método analítico do malation por CLAE}

A curva analítica obtida após a injeção dos padrões de 0,$05 ; 0,10 ; 0,25 ; 0,50 ; 0,75$ e $1,00 \mu \mathrm{g} \mathrm{mL}^{-1}$ de malation encontra-se na figura 1 . O coeficiente de linearidade obtido $\left(\mathrm{R}^{2}\right)$ foi de 0,999 , indicando uma correlação fortíssima entre as variáveis.

O tempo de retenção do analito foi de 4,2 minutos e o tempo de corrida cromatográfica, de 10 minutos para água e 20 minutos para solo.

O método analítico foi específico para malation em solo e água. O limite de detecção calculado foi de $0,017 \mu \mathrm{g} \mathrm{mL}^{-1}$ e o limite de quantificação, $0,051 \mu \mathrm{g} \mathrm{mL}^{-1}$.

Muitos métodos analíticos são descritos para análise de malation por cromatografia gasosa e para análise de múltiplos resíduos (Bernal et al., 1996; Abu-Qare e Abou-Donia, 2001) e requerem um pré-tratamento das amostras, o que ocasiona um encarecimento do processo de extração.

O método proposto neste estudo é simples, utiliza modo isocrático no sistema de CLAE e dispensa o tratamento complexo das amostras, sem provocar prejuízos às análises cromatográficas, oferecendo uma vantagem metodológica e financeira em relação aos métodos normalmente descritos. 


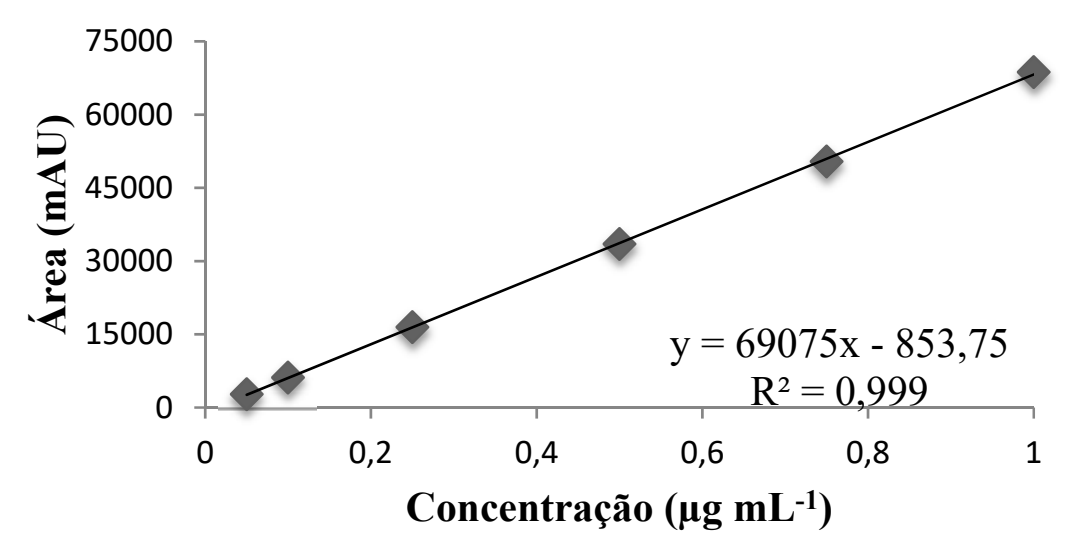

Figura 1. Curva analítica obtida para o inseticida malation com as concentrações de $0,05-1,00 \mu \mathrm{g} \mathrm{mL}^{-1}$.

A redução do tempo da corrida das amostras também é vantajosa quando comparada a métodos multirresíduos. Tahir et al. (2010) obtiveram um tempo de corrida de 28,5 minutos para separação de malation e clorpirifós de amostras de solo por cromatografia gasosa acoplada a detector de nitrogênio e fósforo, com tempo de retenção $\left(t_{R}\right)$ de 18,9 minutos para o inseticida. Morales et al. (2012) analisaram diferentes agrotóxicos em amostras de águas superficiais de regiões agrícolas por CLAE com detector de arranjo de diodos. A duração da corrida cromatográfica do método desenvolvido pelos autores foi de 115 minutos.

Este tempo foi bastante reduzido de acordo com a metodologia aqui proposta, com 10 minutos de corrida cromatográfica para amostras de água e 20 minutos para solo, com tr de 4,2 minutos para o inseticida em ambas as matrizes.

Abu-Qare e Abou-Donia (2001) obtiveram um limite de detecção e quantificação de 0,050 e $0,10 \mu \mathrm{g} \mathrm{mL}^{-1}$ para o malation em metodologia multirresíduos desenvolvida para análise de amostras de plasma e urina humana em CLAE, calculados na proporção de 1:3 e 1:10 do ruído da linha de base. Estes valores são maiores quando comparados com aqueles encontrados neste trabalho $\left(\mathrm{LD}=0,017 \mu \mathrm{g} \mathrm{mL} \mathrm{L}^{-1} \mathrm{e} \mathrm{LQ}=0,051 \mu \mathrm{g} \mathrm{mL}^{-1}\right)$.

\subsection{Método de Extração do Malation das Matrizes de Água e Solo}

As porcentagens de recuperação do malation em solo e água após extração e préconcentração encontram-se na Tabela 2. Todos os valores foram enquadrados na faixa recomendada pela AOAC (1998) para os níveis de fortificação investigados.

Tabela 2. Porcentagem de recuperação do malation em água e solo para três níveis de concentração.

\begin{tabular}{|c|c|c|c|c|c|c|}
\hline \multirow[b]{2}{*}{$\begin{array}{c}\text { Concentração } \\
\left({ }^{\mathrm{a}} \mathrm{mg} \mathrm{L}^{-1} /{ }^{\mathrm{b}} \mathrm{mg} \mathrm{kg}^{-1}\right)\end{array}$} & \multicolumn{3}{|c|}{ Água ${ }^{a}$} & \multicolumn{3}{|c|}{ Solo $^{b}$} \\
\hline & $\begin{array}{c}\% \\
\text { Recuperação }\end{array}$ & $\mathrm{DP}^{1}$ & $\mathrm{CV}^{2}$ & $\begin{array}{c}\% \\
\text { Recuperação }\end{array}$ & $\mathrm{DP}^{1}$ & $\mathrm{CV}^{2}$ \\
\hline 0,005 & 99 & 1,29 & 1,31 & 100 & 8,85 & 8,83 \\
\hline 0,050 & 97 & 0,82 & 0,85 & 94 & 5,88 & 6,27 \\
\hline 0,100 & 95 & 0,24 & 0,27 & 95 & 5,49 & 5,85 \\
\hline Média & 97 & & & 96 & & \\
\hline
\end{tabular}

Nota: ${ }^{1}=$ desvio padrão, $\mathrm{e}^{2}=$ coeficiente de variação $(\%),{ }^{\mathrm{a}}=\mathrm{mg} \mathrm{L}^{-1}$ para água, $\mathrm{e}^{\mathrm{b}}=\mathrm{mg} \mathrm{kg}^{-1}$ para solo. 
A exatidão expressa pelo fator de recuperação, e a precisão expressa pelo coeficiente de variação (CV) estão de acordo com o recomendado pela ANVISA (2003) e ABNT (2005), bem como os outros parâmetros já discutidos anteriormente, garantindo a confiabilidade dos resultados de análise.

A recuperação obtida para malation por Tahir et al. (2010) em seis réplicas de solo proveniente de um campo de golfe fortificado com $10 \mu \mathrm{g} \mathrm{g}^{-1}$ do inseticida foi de 88,5 a 99,9\%, utilizando o método de soxhlet e pré-concentração em rotoevaporador. De acordo com Karyab et al. (2013), a recuperação de malation em amostras de água variou de 45 a 63\%, com microextração em banho ultrassônico utilizando tolueno. Estes valores de recuperação são inferiores aos obtidos neste estudo para ambas as matrizes, indicando a eficiência da metodologia descrita para a extração do inseticida.

De acordo com a especificidade, a linearidade, o limite de detecção e quantificação, a precisão e a exatidão calculados e através das porcentagens de recuperação obtidas nos três níveis de estudo, o método é considerado adequado para análise de malation em amostras de água e solo argiloso.

\section{CONCLUSÃO}

A validação do método cromatográfico desenvolvido neste estudo atende às exigências da Resolução 899/2003 (2003) e da NBR14029 (ABNT, 2005), e garante a confiabilidade dos resultados. A recuperação média do malation obtida após os processos de extração e pré-concentração desenvolvidos para as matrizes água e solo é de 97 e 96\%, respectivamente, e estão enquadradas na faixa recomendada pela AOAC (1998) para os níveis de concentração de estudo.

Este método apresenta vantagens em relação a outros descritos para determinação do malation em amostras de água e solo, principalmente em relação ao procedimento de extração, tempo de análise e limites de detecção e quantificação, além de ser facilmente executado e de gerar dados satisfatoriamente interpretáveis. A aplicação da metodologia aqui proposta às amostras ambientais de água e solo possibilitará uma compreensão do estudo deste contaminante nestas matrizes, mostrando-se uma importante ferramenta na gestão da saúde ambiental.

\section{REFERÊNCIAS}

ASSOCIAÇÃO BRASILEIRA DE NORMAS TÉCNICAS - ABNT. Nbr 13073: limpeza de vidrarias para uso de ensaios de produtos agrotóxicos e afins. Rio de Janeiro, 2003.

ASSOCIAÇÃO BRASILEIRA DE NORMAS TÉCNICAS - ABNT. Nbr 14029: agrotóxico e afins - validação de métodos analíticos. Rio de Janeiro, 2005.

ABU-QARE, A. W.; ABOU-DONIA, M. B. Simultaneous determination of malathion, permethrin, DEET (N,N-diethyl-m toluamide), and their metabolites in rat plasma and urine using high performance liquid chromatography. Journal of Pharmaceutical and Biomedical Analysis, v. 26, n. 2, p. 291-299, 2001. http://dx.doi.org/10.1016/S07317085(01)00407-1

AMARANTE Jr, O. P.; SANTOS, T. C. R.; BRITO, N. M.; RIBEIRO, M. L. Métodos de extração e determinação do Herbicida Glifosato: Breve Revisão. Química Nova, v. 25, n. 3, p. 420-428, 2002. http://dx.doi.org/10.1590/S0100-40422002000300015

AGÊNCIA NACIONAL DE VIGILÂNCIA SANITÁRIA (Brasil) - ANVISA. Resolução RE No 899, de 29 de março de 2003. Diário Oficial [da] União, 02 jun. 2003. 
ASSOCIATION OF OFFICIAL ANALYTICAL CHEMISTS - AOAC. Peer-Verified Methods Program, Manual on Policies and Procedures. Gaithersburg, 1998.

BERNAL, J. L.; NOZAL, M. J.; MARTIN, M. T.; JIMÉNEZ, J. J. Possibilities of gas chromatography-atomic emission detection in pesticide multiresidue analysis Application to herbicide analysis in soils. Journal of Chromatography A, v. 754, n. 1-2, p. 245-256, 1996. http://dx.doi.org/10.1016/S0021-9673(96)00200-2

BRASIL. Departamento de Vigilância Epidemiológica. Diretrizes nacionais para prevenção e controle de epidemias de dengue - Série A. Brasília, 2009. 160 p.

BRITO, N. M.; AMARANTE Jr., O. P.; POLESE, L.; RIBEIRO, M. L. validação de métodos analíticos: estratégia e discussão. Pesticidas: Revista de Ecotoxicologia e Meio Ambiente, v. 13, p. 129-146, 2003. http://dx.doi.org/10.5380/pes.v13i0.3173

CHAIM, A. Processos de aplicação de produtos fitossanitários e contaminação ambiental. Circular Técnica no 3. Jaguariúna: EMBRAPA; CNPDA, 1989.

KARYAB, H.; MAHVI, A. H.; NAZMARA, S.; BAHOJB, A. Determination of water sources contamination to diazinon and malathion and spatial pollution patterns in Qazvin, Iran. Bulletin of Environmental Contamination and Toxicology, v. 90, p. 126-131, 2013. http://dx.doi.org/10.1007/s00128-012-0880-8

KULLURU, P. P.; DAS, B. S.; PANDA, R. K. Evaluation of Sorption and Leaching Potential of Malathion and Atrazine in Agricultural Soils of India. International Journal of Environmental Research, v.4, n. 1, p. 75-90, 2010. http://hdl.handle.net/1807/4949

MORALES, Y. M.; GARCIA, M. F.; QUINTERO, A. B.; DIAZ, P. B.; CONTRERAS, L. M. Niveles de plaguicidas en aguas superficiales de una región agrícola del estado Mérida, Venezuela, entre 2008 y 2010. Revista Internacional de Contaminación Ambiental, v. 28, n. 4, p. 289-301, 2012.

TAHIR, N. M.; SOON, K. H.; ARIFFIN, M. M.; SURATMAN, S. Chlorpyrifos and malathion residues in soils of a terengganu golf course: a case study. The Malaysian Journal of Analytical Sciences, v. 14, n. 2, p. 82-87, 2010. 